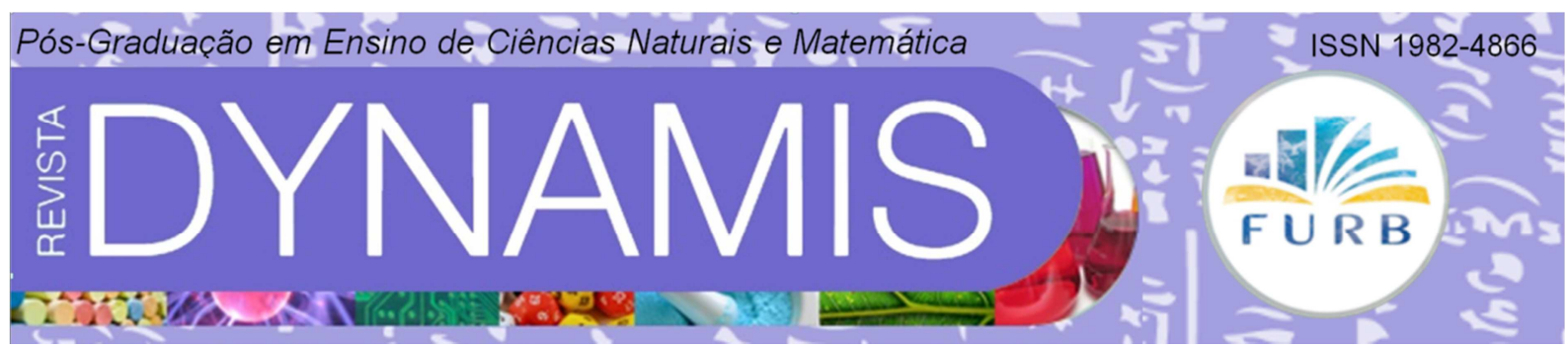

\title{
METODOLOGIA ALTERNATIVA NO PROCESSO DE ENSINO APRENDIZAGEM SOBRE EMBRIOLOGIA E REPRODUÇÃO HUMANA PARA ALUNOS DO ENSINO MÉDIO DAS ESCOLAS DE BLUMENAU
}

Alternative methodology on the learning process about embryology and human reproduction to high school students of Blumenau schools

Débora Baratto de Albuquerque

deborabaratto@gmail.com

Ana Luiza Cim Ribeiro de Souza

analuiza_crs11@hotmail.com

Rúbia Tábata Rigatti

rubiarigatti@hotmail.com

Clarissa Novello Batzner

clarissa.nbatzner@gmail.com

Beatriz Bandeira de Andrade

andrade.beatriz@hotmail.com

Isabela Yones Nogara

isy_nogara@hotmail.com

Paula Prada Radtke

paulinhapr@terra.com.br

Débora Delwing Dal Magro

deboradelwing@yahoo.com.br

Cláudia Almeida Coelho de Albuquerque

claudia@furb.br

Sara Cristiane Barauna

sbarauna@yahoo.com.br

Universidade Regional de Blumenau 


\title{
Resumo
}

A inovação das metodologias de ensino durante o período escolar é de extrema importância para melhoria do conhecimento e do interesse por parte dos alunos acerca dos assuntos de embriologia e reprodução humana. No presente estudo foram coletadas informações referentes a idade e sexo de cada aluno participante da palestra. Também foi aplicado um questionário composto por perguntas acerca dos assuntos Embriologia e Reprodução Humana, previamente à palestra e após a mesma. Nas palestras foram utilizados materiais didáticos maquetes, vídeos, fetos para exposição -, juntamente com a explicação por parte das bolsistas e voluntárias do projeto de extensão, a respeito dos assuntos já citados. Dos 426 alunos que participaram da coleta de dados, 50,23\% eram do sexo feminino e 46,47\% do sexo masculino. Quanto à idade, o estudo abrangeu jovens de 14 a 19 anos, sendo que idade média dos participantes foi de 15,86 anos. A média obtida pelos alunos antes da realização das palestras foi de 8,23 acertos, subindo para 9,91 acertos após a realização das palestras, um aumento correspondente a $20,41 \%$. Com a utilização de um método expositivo alternativo, comprovouse uma melhoria no nível de informação sobre os temas Embriologia e Reprodução Humana por parte dos escolares avaliados, o que sugere uma mudança comportamental devido às palestras educativas. A continuação das exposições nas escolas é imprescindível para a continuidade da ampliação do conhecimento por parte, não somente dos alunos ouvintes, como também da comunidade em geral.

Palavras-chave: Educação. Metodologias alternativas. Reprodução Humana.

\begin{abstract}
The innovation of teaching methods for school attendance is extremely important for improving knowledge and interest of students about the topics presented. In the present study were collected information on age and sex of each student. Additionally, a questionnaire was applied consisting of questions about the issues and Human Embryology and Reproduction, prior to the lecture and after. In the lectures were used teaching materials - models, videos, fetuses for display - along with the explanation on the part of scholars and volunteers of the extension project, about the issues previously mentioned. Of the 426 students who participated in the data collection, $50.23 \%$ were female and $46.47 \%$ male. Regarding age, the study involving the undergraduated from 14 to 19 years, with a mean age of participants was 15.86 years. The average achieved by the students prior to the lectures was 8.23 hits, rising to 9.91 hits after the it, a corresponding increase to $20.41 \%$. The accomplishment of the exhibition and illustrated talk has proven to be an improvement in the level of information on the themes and Human Embryology and Reproduction by schoolchildren, suggesting a behavioral change due to educational lectures. The continuation of exhibitions in schools is essential for the continued expansion of knowledge on the part not only of present students, as well as the hole community.
\end{abstract}

Keywords:Education. Alternative methodologie. Human Reproduction. 


\section{INTRODUÇÃO}

Atualmente, apesar do elevado desenvolvimento sociocultural e tecnológico atingido pela população mundial, de forma generalizada, o conhecimento acerca dos aspectos de crescimento e desenvolvimento biopsicossocial e sexual, tão necessários à construção da identidade psicossocial, não têm alcançado de forma ampla e adequada a maior parte dos adolescentes, ocasionando entre estes altos índices de desinformação sobre diferentes aspectos. Conhecimentos sobre sexualidade e suas respectivas consequências na vida do ser humano, normalmente são negligenciadas tanto pela família dos adolescentes quanto pelas instituições educativas a quem estão submetidos (GOMES et al., 2002). A adolescência compreende o período dos dez aos dezenove anos de idade, conforme a Organização Mundial de Saúde (2004). Atualmente, este fenômeno é entendido tendo em vista uma multiplicidade de categorias sociais diretamente implicadas no processo de constituição do sujeito. (MAHEIRIEET al., 2005).

A sexualidade é um fenômeno da existência humana, portanto, faz parte também da vida dos adolescentes. Entre a população brasileira, o início da vida sexual, ocorre antes dos 15 anos de idade e, o início ainda mais precoce pode ser observado entre mulheres de determinadas regiões do país (SZWARCWALD et al., 2010). É objeto de estudo e intervenção das políticas públicas e tem sido cada vez mais discutida, principalmente devido ao aumento dos índices de gravidez e de incidência de AIDS e outras doenças sexualmente transmissíveis na população jovem (MAHEIRIE et al., 2005). Análises brasileiras revelam que apenas um terço dos jovens ou menos usam preservativo sempre. Segundo dados de pesquisas divulgados pelo Ministério da Saúde (MS), os mais baixos índices de uso se encontram em jovens com idade entre 15 e 19 anos. Outros estudos do Ministério da Saúde Secretaria de Vigilância em Saúde (BRASIL, 2011) revelaram que cerca de 19,3\% das crianças nascidas vivas em 2010 no Brasil são filhos e filhas de mulheres de 19 anos ou menos.

Tendo em vista que a educação é um dos pilares da existência do progresso e da expansão de uma sociedade, a mesma expressa, através da visão clássica dos trabalhos de Durkheim (1973), uma doutrina pedagógica que se apóia na concepção do homem e sociedade; ou seja, o processo educacional emerge através da família, igreja, escola e comunidade (NOÉ, 2000). A educação sexual dos jovens é de responsabilidade prioritária da família, porém a sociedade e a escola participam deste processo (JARDIM, 2006). De acordo com um estudo realizado por Jardim (2006), os professores de escolas do município de Jandira - SP não possuem conhecimento suficiente para auxiliar no processo de educação sexual dos adolescentes, necessitando de programas de treinamento para os professores e palestras educativas aos alunos.

O ensino de ciências tem um importante papel na formação de crianças e adolescentes, e não deve centrar-se apenas na aprendizagem passiva de conceitos. Entretanto, a transmissão, na escola, de informações destituídas de significado é possivelmente uma das causas do desinteresse pelas aulas e pelo que nelas precisa ser aprendido. Os estudantes precisam utilizar os conhecimentos científicos como instrumentos que ofereçam novos significados e percepções sobre o mundo, criando outras possibilidades de interação com a realidade. É necessário ensinar de forma criativa e estimulante, sem abdicar o rigor do que significa aprender ciência. Teremos uma sociedade melhor quando acontecerem transformações na educação, principalmente transformações relacionadas à formação dos professores. Frente a 
estes desafios, bem como ao incremento de ações que aproximassem a Universidade das escolas, foi concebido na Universidade Regional de Blumenau (FURB) o Programa de Extensão "Educação em Ciências para o Século XXI", que atualmente se encontra em sua terceira edição. O programa tem o objetivo de apoiar Clubes de Ciências e atuar na formação continuada de professores, bem como fornecer materiais de apoio para as aulas de ciências das escolas da rede pública de Blumenau.

Em 2012, buscando um objetivo em comum ao Programa de Extensão "Educação em Ciências para o Século XXI", foi desenvolvido o Projeto de Extensão "Educação em Embriologia e Reprodução Humana".Este projeto foi pensado após a análise de dados sobre o elevado índice de adolescentes grávidas no país, que de acordo com o Ministério da Saúde, totalizou 536 mil casos no ano de 2012 e à falta de conhecimento sobre desenvolvimento embrionário, reprodução e sexualidade dos acadêmicos que ingressam na FURB.O projeto visa o desenvolvimento de materiais didático-pedagógicos de maneira a transmitir o conhecimento sobre Embriologia e Reprodução Humana através de métodos atrativos como a construção de maquetes, jogos, cartazes explicativos e palestras com a utilização de peças anatômicas de fetos. O projeto tem como foco, além de levar o conhecimento para adolescentes do ensino médio das escolas de Blumenau - SC, estreitar ainda mais os laços entre a Universidade e a comunidade, bem como capacitar profissionais e acadêmicos da área da saúde e educação.

Analisando dados coletados através da execução do Projeto de extensão "Educação em Embriologia e Reprodução Humana", o objetivo do presente trabalho foi de verificar se houve aprimoramento do nível de conhecimento, por parte de estudantes de escolas públicas e particulares de Blumenau - SC, sobre Embriologia e Reprodução Humana, através de palestras educativas e metodologias de ensino não convencionais, como o método expositivo.Este método consiste, segundo Rangel (2007), "na exposição oral estruturada de uma temática de estudo". Apresenta como princípios essenciais o domínio dos conteúdos a serem apresentados e a correta maneira de exposição dos mesmos, juntamente com a prática de perguntas, respostas e diálogos entre estudante e professor.

\section{PROCEDIMENTOS METODOLÓGICOS}

Os locais de pesquisa foram quatro escolas, selecionadas de maneira aleatória, da cidade de Blumenau - SC, sendo que duas escolas fazem parte da rede particular de ensino e, as outras duas, da rede pública. A população-alvo foi composta por alunos do Ensino Médio das escolas propostas totalizando 426 alunos, sendo 257 destes alunos de escolas públicas e o restante, 169 alunos, de escolas particulares.

Para a realização do estudo foram agendadas palestras nas escolas supracitadas, durante o período de março a novembro de 2014. Cada turma assistiu a uma palestra, sendo esta conduzida por uma dupla de acadêmicas do curso de medicina da FURB e participantes do Projeto de Extensão "Educação em Embriologia e Reprodução Humana". As palestrantes receberam treinamento prévio teórico e prático, através de teste em estudo piloto antes da realização das palestras. Cada palestra teve duração de duas horas e abordou os seguintes temas: sistemas reprodutor masculino e feminino, fecundação, etapas do desenvolvimento fetal, métodos contraceptivos e doenças sexualmente transmissíveis. 
Primeiramente foram explicados aos alunos os sistemas reprodutor feminino e masculino com o uso de maquetes e imagens para facilitar o entendimento dos adolescentes. Posteriormente, os alunos assistiram a um vídeo com música elaborado por estudantes de medicina da FURB explicando sobre o processo de fecundação. Em seguida, fetos do $3^{\circ}, 5^{\circ} \mathrm{e}$ $6^{\circ}$ mês de gestação do Laboratório de Anatomia da FURB foram expostos para a visualização com explicação do desenvolvimento fetal conforme os meses de gestação. Os alunos foram orientados e autorizados a fazer questionamentos e apresentar dúvidas pessoais sobre os temas. Os materiais utilizados na apresentação, incluindo maquetes dos sistemas reprodutor masculino e feminino, uma amostra em tamanho aumentado do ovócito feminino, os núcleos que formam o zigoto e os fetos, foram confeccionados e/ou catalogados no ano de 2013 pelas participantes do projeto, servindo como material didático na demonstração das palestras, melhorando o processo de ensino-aprendizagem.

Para abordar os métodos contraceptivos, foi exposta uma amostra de cada método. Explicou-se a forma de uso e foram citadas as vantagens e desvantagens de cada um. Explanaram-se os seguintes métodos: preservativo masculino e feminino, diafragma, pílula anticoncepcional, pílula de emergência (pílula do dia seguinte), dispositivo intrauterino (DIU), anticoncepcional injetável e adesivo e anel vaginal. Também foram explicados os métodos popularmente conhecidos como "coito interrompido" e "tabelinha". Ao fim de cada palestra foi realizada uma dinâmica envolvendo o tema de doenças sexualmente transmissíveis e proteção. Nessa dinâmica, cada aluno recebeu um copo com água. Aleatoriamente e sem o conhecimento dos adolescentes, colocou-se hidróxido de sódio $(\mathrm{NaOH})$ em alguns copos, representando a contaminação destes por alguma doença sexualmente transmissível. Orientou-se que cada aluno realizasse a troca de parte do líquido de seu copo com alguns colegas, representando, dessa forma, a troca de fluidos corporais que ocorre nas relações sexuais desprotegidas. Ao fim da dinâmica, foram adicionadas algumas gotas de fenolftaleína em todos os copos e este, ao reagir com o hidróxido de sódio, concebeu uma coloração rósea ao líquido, comprovando a contaminação. Ao perceber o número final de "contaminações", foi enfatizado aos alunos que a transmissão dessas doenças ocorre independentemente de raça ou sexo, muitas vezes até sem o conhecimento do portador e, assim, demonstrou-se a importância da proteção durante o ato sexual e, por consequência, da prevenção de doenças.

As fontes de pesquisa utilizadas para a obtenção de informações científicas para serem expostas nas palestras foram as mais variadas. Dentre elas, artigos, livros acadêmicos e livros de Ensino Médioforam utilizados para o planejamento das informações para levá-las aos jovens e melhor entendimento do aprendizado dos mesmos.A capacitação das acadêmicas participantes do projeto ocorreu desde o início das atividades, na busca de materiais didáticos usados pelos estudantes de ensino médio e na orientação dos palestrantes pelos professores e preceptores responsáveis pelo projeto no que diz respeito às metodologias de ensino que poderiam ser usadas na realização das palestras. A comunicação com jovens e adolescentes pode ser um obstáculo frente à inexperiência dos oradores ao ministrar as aulas sobre assuntos complexos expostos nas mesmas - Embriologia e Reprodução Humana. A produção de materiais didáticos, como maquetes, vídeos e cartazes, foi de suma importância na superação deste obstáculo, para auxiliar no entendimento dos assuntos supracitados.

Antes e após a realização das palestras cada aluno respondeu a um mesmo questionário de forma anônima, com 17 perguntas de múltipla escolha sobre os temas propostos, elaborados e analisados pelos participantes do projeto e seus orientadores e, assim, 
foi observada a evolução do aprendizado dos adolescentes. Participaram da palestra e da aplicação dos questionários apenas os alunos que possuíam autorização dos pais e/ou responsáveis, por termo previamente entregue e assinado pelos mesmos. Os critérios de exclusão do estudo foram: alunos que não possuíam autorização dos responsáveis e alunos que não compareceram à aula agendada. Posteriormente, fez-se a análise dos questionários preenchidos pelos alunos. Foi calculado o número de acertos de cada questionário e, então, a média, antes e após a realização da palestra. Os dados foram analisados pelo teste do QuiQuadrado, sendo consideradas significativas as diferenças quando o valor de $\mathrm{p}<0,05$.

\section{ANÁLISE E DISCUSSÃO DOS RESULTADOS}

Dos 426 alunos que participaram da coleta de dados, 50,23\% eram do sexo feminino e $46,47 \%$ do sexo masculino, representando equiparidade de gêneros (Tabela 1). Quanto à idade, o estudo abrangeu jovens de 14 a 19 anos, sendo mais prevalentes as de 15 anos (28,8\%), 16 anos $(27,9 \%)$ e 17 anos $(25,1 \%)$, as quais representam as idades médias dos estudantes que frequentam o Ensino Médio. A idade média dos participantes foi de 15,86 anos (Tabela 2).

Tabela 1- Distribuição dos participantes segundo o sexo.

\begin{tabular}{ccc}
\hline SEXO & NÚMERO DE ALUNOS & PORCENTAGEM \\
\hline Feminino & 214 & $50,23 \%$ \\
Masculino & 198 & $46,47 \%$ \\
Não respondeu & 14 & $3,30 \%$ \\
\hline
\end{tabular}

Fonte: Dados da pesquisa

Tabela 2 - Distribuição dos participantes segundo a idade.

\begin{tabular}{ccc}
\hline IDADE (ANOS) & NÚMERO DE ALUNOS & POCENTAGEM (\%) \\
\hline 14 & 42 & $9,86 \%$ \\
15 & 123 & $28,87 \%$ \\
16 & 119 & $27,93 \%$ \\
18 & 107 & $25,11 \%$ \\
19 & 16 & $3,75 \%$ \\
Não respondeu & 4 & $0,94 \%$ \\
\hline
\end{tabular}

Fonte: Dados da pesquisa 
A população brasileira sofre a iniciação sexual ainda muito jovem - em torno de 15 anos de idade. Frente a este fenômeno, o estudo focalizou nos estudantes de Ensino Médio para, justamente, informá-los sobre questões que cercam a sexualidade e suas consequências, uma vez que, de maneira geral, o adolescente não recebe pela família informações que envolvam a saúde e, quando tem acesso, essas informações são muitas vezes limitadas e inadequadas, provenientes de amigos, de pessoas pouco preparadas para essa função (GOMES et al., 2002).

Observamos um avanço no conhecimento dos alunos do Ensino Médio das escolas públicas e particulares de Blumenau sobre Embriologia e Reprodução Humana, uma vez que a média de acertos das questões do questionário específico aumentou 20,41\%. A média obtida pelos alunos antes da realização das palestras foi de 8,23 acertos, subindo para 9,91 acertos após a realização das palestras. (Figura 1).

Figura 1 - Resultados obtidos com a aplicação do questionário específico sobre Embriologia e Reprodução Humana antes e depois da realização da palestra

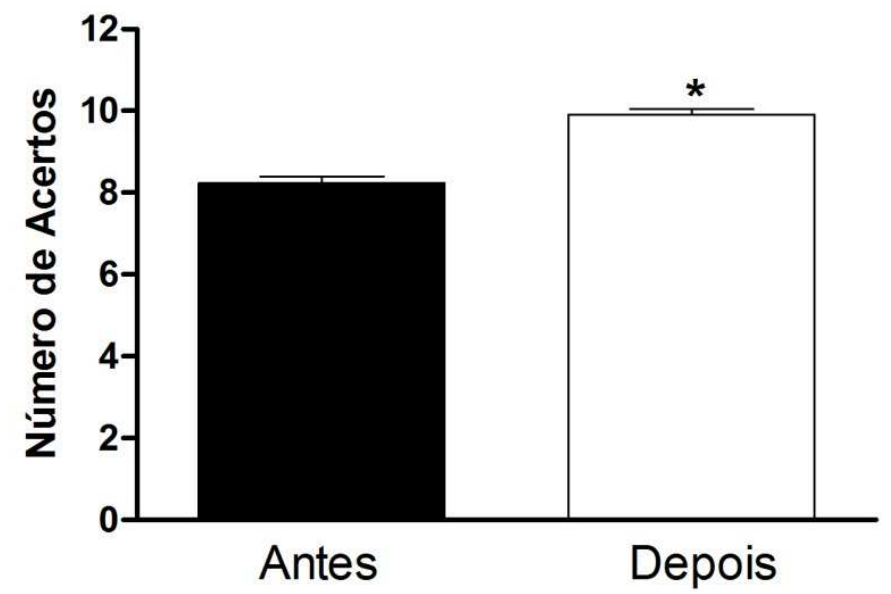

- Os resultados representam a média de acertos \pm erro padrão. *p<0,05 em relação aos resultados antes da palestra.

Fonte: Dados da pesquisa

A partir do resultado obtido, podemos inferir que a utilização de um método expositivo alternativo, usado para a apresentação das palestras, demonstrou considerável eficácia. $\mathrm{O}$ método expositivo utilizado consiste na comunicação verbal por parte do orador em forma de narração ou demonstração. O domínio do palestrante a respeito do assunto e sua maneira de transmiti-lo aos ouvintes, juntamente com o uso de materiais didáticos expositivos, são a base deste método (RANGEL, 2007).Segundo um estudo feito no estado de São Paulo, o projeto educativo realizado, que ocorreu através de palestras expositivas e materiais didáticos semelhantes aos usados neste estudo, demonstrou ótimos resultados (GENARI et al., 2012). Chiaravalloti Neto et al. (1998) ao avaliarem os resultados de um projeto educativo em duas fases, no Município de São José do Rio Preto, SP, verificaram que as atividades educativas geraram ganhos de conhecimento da população, assim como no estudo de Uchoaet al. (2004), ao avaliarem a aquisição de conhecimento por parte dos 
escolares do Ensino Fundamental no município de Maricá, MG, por meio de palestras, cartazes e cartilhas.

A contextualização, promovida pelos palestrantes durante a exposição, resulta em aprendizagens significativas recíprocas entre aluno e objeto do conhecimento, excedendo o âmbito conceitual, sendo assim qualificada como estratégia metodológica para a compreensão de fatos ou situações do cotidiano dos alunos. Contextualizar deve facilitar o processo de ensino aprendizagem, buscar contato com o tema e criar o interesse pelo conhecimento com aproximações entre conceitos biológicos e vida do indivíduo, pois estabelece analogia entre o conteúdo da educação formal ministrado em sala de aula e cotidiano do educando (SCAFI, 2010).

Em suma, através do discurso realizado e utilizando materiais expositivos, quando associada ao método clássico de ensino, como durante as aulas ministradas em escolas públicas e particulares, Faculdades e Universidades de todo o país, apresenta resultados positivos na complementação do ensino básico (PLIESSNIG; KOVALICZN, 2009). Além de proporcionar um incremento no nível de conhecimento dos estudantes, as palestras realizadas por meio deste método provocam o mesmo efeito nos seus respectivos familiares e amigos, representando um veículo de informações para a sociedade como um todo. O uso de metodologias alternativas propõe um processo ensino aprendizagem mais atrativo para os alunos, provocando questionamentos e interesse por parte dos mesmos.

Através das palestras educativas foi conseguido aproximar a Universidade da comunidade de forma que, tanto os alunos participantes das mesmas quanto seus amigos e familiares, puderam compreender o universo de uma instituição de ensino superior e instigálos a prosseguir com seus estudos. Essa proximidade proporcionada pelo projeto trouxe uma ampliação da associação entre os mesmos, fazendo com que os alunos e professores das escolas membro viessem até os palestrantes para a retirada de dúvidas referentes aos assuntos mostrados. Outro objetivo alcançado foi o da elaboração de materiais didático-pedagógicos com ênfase aos aspectos relacionados ao desenvolvimento embrionário e anexos.

\section{CONSIDERAÇÕES FINAIS}

Observou-se, por meio desse Projeto, que houve um aumento geral do nível de conhecimento dos estudantes participantes, tanto de escolas públicas quanto de particulares, sobre Embriologia e Reprodução Humana. Com esse resultado, comprova-se a eficácia do método expositivo de ensino, uma metodologia educacional não convencional, sendo caracterizado pelo diálogo e comunicação constantes entre discente e docente, bem como pela apresentação de materiais de exposição. Esse método de ensino proporciona não apenas um incremento no nível de conhecimento dos estudantes, mas também de seus familiares e de amigos, sendo considerado, portanto, um veículo de informações para a sociedade em geral. Proporciona, também, uma maior aproximação entre professores e alunos, havendo, assim, melhor compreensão dos aspectos sociais e cotidianos de ambas as partes.

Com os resultados obtidos, faz-se necessária a ampliação do projeto para escolas de todo o país, de modo a aprimorar o conhecimento em busca de novos resultados e futuros benefícios. Fortalece-se assim a necessidade de ações inovadoras voltadas para a educação sexual de adolescentes. A abordagem e a dinâmica desenvolvida para o estudo em questão demonstrou-se positiva, criando uma empatia e permitindo um diálogo claro e desinibido entre palestrantes e alunos, de modo que se tornou possível conhecer e elucidar as dúvidas 
constantes que rondam a mente desta faixa etária.

\section{REFERÊNCIAS}

BIBLIOTECA VIRTUAL EM SAÚDE DO MINISTÉRIO DA SAÚDE. Adolescência sem filhos é bem melhor. Secretaria Municipal de Saúde de Palmas. Disponível em: <http://bvsms.saude.gov.br/gravidez-na-adolescencia/>. Acesso em 30 jan. 2015

CHIARAVALLOTI, N. F.; MORAES, M. S.; FERNANDES, M. A. Avaliação dos resultados de atividades de incentivo à participação da comunidade no controle da dengue em um bairro periférico do Município de São José do Rio Preto, São Paulo, e da relação entre conhecimentos e práticas desta população. Cadernos de Saúde Pública, São Paulo, v.14 supl. 2, p. 101-1, 1998.

DURKHEIM, E. Educación y Sóciología: textos e intervenciones de los sociólogos clásicos.Buenos Aires: Editorial Shapire, 1973.

ESCOLA DE SAÚDE PÚBLICA DE MINAS GERAIS. Técnicas de Ensino. Disponível em: http://ead.esp.mg.gov.br/nte/docs/tecnicasensino_top4.pdf. Acesso em 19 jan., 2015.

GENARI, I. C. C.. Atividades de educação em saúde sobre leishmaniose visceral para escolares. Veterinária e Zootecnia, v.19, n.1, p.99-107, 2012.

GOMES, W. A. et al. Nível de informação sobre adolescência, puberdade e sexualidade entre adolescentes. Jornal de Pediatria, v. 78, n. 4, p. 301-8, 2002.

JARDIM, D. P.. Orientação sexual na escola: a concepção dos professores de Jandira - SP. Revista Brasileira de Enfermagem, v. 59, n. 2, p. 157-62, 2006.

MAHEIRIE, K. et al..Oficinas sobre sexualidade com adolescentes: um relato de experiência. Psicologia em Estudo, v.10, n.3, p. 537-542, 2005.

BRASIL. Ministério da Saúde. Preservativo masculino. Brasília: Secretaria de Assistência à Saúde, 2000.

BRASIL. Ministério da Saúde. Saúde Brasil: uma análise da situação de saúde e a vigilância da saúde da mulher. Brasília: Editora MS, 2011.

NOÉ, A.. A Relação Educação e Sociedade: Os fatores sociais que interferem no processo educativo. Avaliação, Campinas, v. 5, n.3, p.21-26, 2000.

ORGANIZAÇÃO MUNDIAL DA SAÚDE. A Saúde dos Jovens: Documento de trabalho para discussões técnicas. Genebra, 1986.

PLIESSNIG, A. F. ; KOVALICZN, R. A.. O uso de metodologias alternativas como forma de superação da abordagem pedagógica tradicional na disciplina de biologia.2009. Disponível em: <http://www.diaadiaeducacao.pr.gov.br/portals/pde/arquivos/1-4.pdf>. Acesso em 10 fev. 2015 . 
Ana Luiza Cim Ribeiro de Souza, Débora Baratto de Albuquerque, Rúbia TábataRigatti, Clarissa NovelloBatzner, Beatriz Bandeira de Andrade, Isabela YonesNogara, Paula Prada Radtke, DéboraDelwing Dal Magro, Cláudia Almeida Coelho de Albuquerque, Sara Cristiane Barauna

RANGEL, M.. Métodos de Ensino para a aprendizagem e dinamização das aulas. Magistério, Formação e Trabalho Pedagógico. São Paulo: Papirus, 2007.

SCAFI, S. H. F.. Contextualização do ensino de química em uma Escola Militar. Química Nova na Escola, v. 32, n. 3, p. 176-183, 2010.

SZWARCWALD, C. L. et al.. Avaliação da adequação das informações de mortalidade e nascidos vivos no Estado de Pernambuco, Brasil. Cadernos de Saúde Pública, v.26, n. 4, p. 671-681, 2010.

UCHOA, C. M. A. et al. Educação em saúde: ensinando a leishmaniose tegumentar americana. Caderno de Saúde Pública, São Paulo, v.20, p. 935-41, 2004. 